Cesar G. Victora 1

Fernando C. Barros 1

Elaine Tomasi 1

Ana Maria Menezes 1

Bernardo L. Horta ${ }^{1}$

Elizabeth Weiderpass 1

Juraci A. Cesar 4

Juvenal S. D. Costa 1

Maria Teresa Olinto 1

Ricardo Halpern 1

Maria del Mar Garcia 3

J. Patrick Vaughan 2

\section{Tendências e diferenciais na saúde materno-infantil: delineamento e metodologia das coortes de 1982 e 1993 de mães e crianças de Pelotas, Rio Grande do Sul}

\author{
Trends and defferentials in maternal and child \\ health: design and metodology of the 1982 and \\ 1993 birth cohort studies in Pelotas, \\ Rio Grande do Sul
}

${ }^{1}$ Departamentos de Medicina Social, MaternoInfantil e de Clínica Médica, Faculdade de Medicina, Universidade Federal de Pelotas. C. P. 464, Pelotas, RS 96001-970, Brasil.

2 Department of Public Health and Policy, London School of Hygiene and Tropical Medicine. Keppel Street, London WCIE 7HT, $U K$.

3 Escuela Andaluza de Salud Publica, Campus

Universitario de Cartuja. Apartado de Correos 2070, Granada 18080, España.

4 Departamento MaternoInfantil, Universidade do Rio Grande. Rua Engenheiro Alfredo Huch, 475. Rio Grande, RS, 96201-460, Brasil.

\begin{abstract}
Important changes have taken place in both the economy and health care in Brazil since 1980 and may have affected maternal and child health. Two studies in the city of Pelotas in Southern Brazil provide a unique opportunity for assessing these issues. Cohorts of mothers and infants from 1982 and 1993 were studied from the time of delivery. In both years, all mothers identified in the city's maternity hospitals answered a standardized questionnaire, and their infants were examined. Over 99\% of all children born in the city in each of the two years were included in the cohorts, totalling 5,914 live births in 1982 and 5,249 in 1993. Deaths among these children were monitored prospectively, as were all hospital admissions in the 1993 cohort. In 1982, attempts were made to locate a 25\% sample of the children at the mean age of 12 months, as well as all of the cohort children at the mean age of 20 months; $82 \%$ of the former were located, and thanks to a change in strategy, 87\% were traced at 20 months. In 1993, a search was made at 12 months of age for $20 \%$ of all children plus all low birthweight infants, and 95\% were successfully traced. This paper presents the main methodological aspects of both cohort studies, the principal results of which are presented in the subsequent articles in this supplement.
\end{abstract}

Key words Epidemiology; Cohort Studies; Maternal Health; Child Health

Resumo Mudanças ocorridas na última década em termos econômicos e assistenciais podem haver afetado a saúde materno-infantil. Dois estudos foram realizados em Pelotas, Rio Grande do Sul. As coortes de mães e recém-nascidos nos anos de 1982 e 1993 foram estudadas desde o nascimento. As mães foram identificadas nos hospitais-maternidade e responderam a um questionário padronizado, sendo seus filhos examinados. Mais de 99\% dos recém-nascidos foram incluídos nas coortes, totalizando 5.914 nascidos vivos em 1982 e 5.249 em 1993. A mortalidade das crianças foi monitorizada, e em 1993 as hospitalizações também o foram. Em 1982, tentouse localizar cerca de 25\% das crianças aos 12 meses de idade e todas com a idade média de vinte meses. Foi possivel encontrar cerca de 82\% das crianças aos 12 meses e, graças a uma mudança de estratégia, $87 \%$ aos vinte meses. Em 1993, tentou-se acompanhar 20\% das crianças e mais todos os recém-nascidos de baixo peso aos 12 meses de idade, sendo 95\% localizados. Este artigo descreve os principais aspectos metodológicos de ambos os estudos, cujos principais resultados estão incluídos nos próximos artigos desse suplemento.

Palavras-chave Epidemiologia; Estudos Longitudinais; Saúde Materna; Saúde da Criança 


\section{Introdução}

O acompanhamento da evolução dos indicadores de saúde materno-infantil é essencial para avaliar o impacto de mudanças sociais e econômicas, assim como de eventuais avanços ou retrocessos na disponibilidade e qualidade dos serviços de saúde. A monitorização deve incluir o estudo das tendências em indicadores de saúde baseados em estatísticas de rotina. Tais estatísticas, no entanto, podem ser afetadas por problemas (Laurenti et al., 1985), como o sub-registro, a cobertura incompleta e a falta de padronização na determinação das causas de óbitos e de hospitalizações. A questão do sub-registro, por exemplo, prejudica o estudo das tendências de mortalidade perinatal devido à freqüente omissão dos óbitos fetais. As estatísticas vitais são também apresentadas de forma agregada, o que impede a verificação de diferenciais de saúde entre, por exemplo, crianças de distintas categorias sócio-econômicas ou de peso ao nascer.

Assim, é essencial subsidiar os estudos de tendências baseados em estatísticas vitais com pesquisas de base populacional, especificamente delineadas para evidenciar diferenciais intrapopulacionais, e que apresentem cuidado metodológico na mensuração e análise dos eventos em estudo. $\mathrm{O}$ presente artigo descreve o delineamento, a metodologia e os principais resultados de dois amplos estudos de coortes de base populacional sobre saúde materno-infantil realizados na cidade de Pelotas, RS, nos anos de 1982 (Victora et al., 1989; Barros et al., 1990; Victora et al., 1996).

No período entre 1982 e 1993, ocorreram no país mudanças paradoxais, tanto em termos sócio-econômicos quanto assistenciais. Por um lado, programas de ajuste econômico foram incriminados como tendo importante impacto sobre a qualidade de vida da população, principalmente de seu segmento mais vulnerável, o materno-infantil (Macedo, 1988). Entre 1980 e 1992, o crescimento anual do Produto Interno Bruto per capita foi estimado em apenas $0,4 \%$ (Grant, 1995). Na cidade de Pelotas, por exemplo, a economia foi marcadamente afetada durante esta década, com o fechamento de diversas indústrias alimentícias que representavam a principal atividade manufatureira no Município.

A década testemunhou também importantes mudanças na qualidade e no acesso aos serviços de saúde por parte da população. Mudanças positivas, como a universalização do atendimento previdenciário e o aumento tanto no número de profissionais de saúde quanto no de unidades sanitárias, foram acompanhadas de uma vasta crise no setor saúde, com o sucateamento das instituições públicas e um crescimento marcado na assistência privada através dos convênios e seguros saúde.

Ao mesmo tempo, o estudo de tendências de mortalidade infantil com base no registro civil, assim como através de técnicas indiretas baseadas nos censos demográficos, mostram importantes declínios em nível nacional, embora os patamares atuais ainda sejam insatisfatórios. O Unicef estima que a mortalidade de menores de cinco anos decresceu em 3\% ao ano, no Brasil, entre 1980 e 1992 (Grant, 1995). Para o Estado do Rio Grande do Sul, o coeficiente de mortalidade infantil caiu de 33,2 por mil nascidos vivos em 1982 para 19,2 em 1993 (SSMA, 1995). Os estudos no nível agregado impedem a constatação dos diferenciais entre os distintos grupos sociais, os quais, no entanto, podem ser evidenciados valendo-se de pesquisas como as descritas no presente artigo.

Os dois estudos longitudinais aqui descritos representam um conjunto único de dados em nosso país. Tanto a coorte de 1982 quanto a de 1993 iniciaram com a identificação, nos hospitais-maternidade de Pelotas, de todas as crianças nascidas de mães residentes na área urbana, totalizando 6.011 nascimentos (5.914 nascidos vivos) em 1982 e 5.304 (5.249 nascidos vivos) em 1993. Ambos os estudos acompanharam prospectivamente amostras destas crianças.

A experiência de 1982 mostrou que as mais importantes alterações na saúde infantil ocorriam durante o primeiro ano de vida, o que orientou o estudo de coorte de 1993 a concentrar-se nesta faixa etária.

Os principais objetivos do estudo comparativo da evolução da saúde materno-infantil foram:

1ㅇ) caracterizar as populações de mães que deram à luz nos hospitais-maternidade da cidade nos anos de 1982 e 1993, em termos sócio-econômicos, demográficos, ambientais e relativos à utilização de serviços de saúde;

2o) descrever diversos indicadores de morbimortalidade, crescimento e desenvolvimento em amostras populacionais de suas crianças;

3o) desagregar os indicadores de saúde e assistenciais de mães e crianças conforme grupos sócio-econômicos, e os indicadores infantis conforme categorias de peso ao nascer;

4o) comparar os indicadores de $1993 \mathrm{com}$ os obtidos em 1982 e interpretar as eventuais modificações de acordo com as mudanças econômicas e assistenciais;

5o) subsidiar o planejamento local e nacional 
de ações de saúde materno-infantil e identificar grupos de risco para intervenções sanitárias.

O presente artigo descreve o delineamento e a metodologia dos dois estudos de coorte, assim como os resultados iniciais referentes ao número de crianças incluídas inicialmente no estudo e as perdas de acompanhamento. Os principais resultados das duas pesquisas encontram-se resumidos nos demais artigos do presente suplemento.

\section{Delineamento e metodologia}

Pelotas é uma cidade de porte médio localizada no extremo sul do país. Sua população urbana aumentou de 214.164 em 1982 para 274.285 em 1993 (dados não publicados, Prefeitura Municipal de Pelotas). As principais atividades agrícolas na região são as plantações de arroz, a fruticultura e a horticultura; há também a atividade pecuária. Na área urbana, o setor de serviços é o mais desenvolvido, com duas Universidades e diversos órgãos públicos. Pelotas é ainda um centro comercial servindo a todo o extremo sul do país. As atividades industriais incluem principalmente o beneficiamento de alimentos (engenhos de arroz, frigoríficos, fábricas de conservas) e a construção civil. Seu produto interno bruto per capita é de quase US\$2.700 (Klering, 1992). Mais de 90\% dos domicílios dispõem de água tratada e quase metade de rede de esgotos (Unicef/IBGE, 1994). O serviço de saúde é oferecido de forma gratuita através de 43 unidades básicas de saúde, cinco ambulatórios especializados e cinco hospitais, havendo um médico para cada 260 habitantes. Apesar dessa infra-estrutura, na periferia da cidade existem bolsões onde se concentra a população de baixa renda, cujos níveis de saúde, como será visto nos demais artigos desse suplemento, deixa bastante a desejar.

Nessa seção, discutem-se os principais aspectos metodológicos dos dois estudos de coorte. Maiores detalhes das metodologias estão disponíveis em outras publicações (Victora et al., 1989; Victora et al., 1996) e nos artigos que se seguem.

\section{Coorte de 1982}

A primeira coorte incluiu todas as crianças nascidas entre 1o de janeiro e 31 de dezembro de 1982 nas quatro maternidades então existentes em Pelotas. As mães foram entrevistadas logo após o parto através de um questionário aplicado por estudantes de Medicina e médi- cos recém-formados, o qual incluía informações sobre fatores sócio-econômicos e demográficos, assim como dados sobre a gestação e suas intercorrências. As crianças e suas mães foram pesadas e medidas na maternidade. Embora a equipe de pesquisa não estivesse presente nas pesagens - que eram realizadas nas 24 horas do dia - as balanças utilizadas foram calibradas regularmente. A renda familiar mensal foi calculada pela soma das rendas individuais e expressa em cinco grupos: $<1,0$ salário mínimo (SM); 1,1 a 3,0 SM; 3,1 a 6,0 SM; 6, 1 a 10,0 SM e >10,0 SM.

A mortalidade das crianças foi monitorizada através de visitas regulares a todos os hospitais, cemitérios, cartórios de Registro Civil e à Delegacia Regional de Saúde, onde os registros de óbito eram centralizados.

As crianças nascidas em 1982 foram acompanhadas em diversas ocasiões. O primeiro acompanhamento realizou-se no início de 1983, incluindo as crianças nascidas de janeiro a abril de 1982, que então apresentavam entre nove e 15 meses de idade (idade média de cerca de 12 meses). Acompanhamentos subseqüentes foram realizados em 1984 (idade média de aproximadamente vinte meses) e 1986 (idade média de cerca de 42 meses). A maior parte dos resultados descritos no presente volume diz respeito aos acompanhamentos de 1983 e 1984. No primeiro, tentou-se localizar as 1.820 crianças nascidas nos quatro primeiros meses de 1982 por intermédio dos endereços fornecidos pelas mães na entrevista perinatal. No segundo, realizou-se um censo, visitando todos os 68.590 domicílios da zona urbana em busca de todas as crianças da coorte que ainda residissem em Pelotas. Os percentuais de perdas de acompanhamento nos dois estudos estão descritos na seção de resultados.

Durante os estudos de acompanhamento, aplicava-se à mãe ou à pessoa responsável pela criança questionários padronizados incluindo perguntas sobre variáveis sócio-econômicas, demográficas, ambientais e referentes a aspectos da dieta, da saúde, do uso de serviços médicos (consultas preventivas, curativas e hospitalizações) e do desenvolvimento da criança. Os questionários eram aplicados por universitários das áreas da saúde e serviço social. As crianças eram pesadas com balança CMS tipo Salter, especialmente projetada para esse tipo de pesquisa domiciliar, e eram medidas deitadas com um infantômetro AHRTAG (Barros \& Victora, 1991). A metodologia de pesagem e mensuração era padronizada (Jelliffe, 1966) e, antes do início de cada fase do trabalho de campo, os entrevistadores foram treinados du- 
rante diversas sessões realizadas em creches. Cerca de 5\% das entrevistas e mensurações eram repetidas por um supervisor para controle de qualidade.

Durante o censo de todos os domicílios urbanos realizado em 1984, tentou-se também identificar crianças nascidas em 1982 de parto domiciliar. Apenas 46 dessas crianças foram encontradas, o que garante a representatividade populacional da coorte hospitalar, por haver incluído cerca de 99,2\% de todos os nascimentos do ano.

\section{Coorte de 1993}

O delineamento do estudo de coorte de 1993 foi mais complexo do que o anterior, havendo incluído cinco subprojetos: perinatal, de acompanhamento, de mortalidade infantil, de hospitalizações e de desenvolvimento psicomotor.

O subprojeto perinatal foi muito similar ao de 1982. As cinco maternidades da cidade foram visitadas diariamente de 1 o de janeiro a 31 de dezembro de 1993, sendo as mães entrevistadas através de um questionário padronizado com perguntas sobre variáveis sócio-econômicas, demográficas, reprodutivas, comportamentais, assistenciais e sobre morbidade. Os recém-nascidos foram pesados ao nascer com balanças pediátricas de mesa com precisão de $10 \mathrm{~g}$, aferidas semanalmente com pesos-padrão. Os recém-nascidos foram examinados pela equipe de pesquisa, sendo seu comprimento medido na posição supina com material padronizado (infantômetros ARTHAG (Barros \& Victora, 1991), e o perímetro cefálico, com fita métrica inelástica, ambos com precisão de 1 $\mathrm{mm}$. A idade gestacional foi avaliada no primeiro dia de vida por meio do método de Dubowitz (Dubowitz et al., 1970). As mães foram pesadas e medidas pela equipe de entrevistadores que incluiu médicos residentes e estudantes de Medicina, previamente treinados nas técnicas de entrevista e mensuração. Cinco por cento das entrevistas e avaliações da idade gestacional foram repetidas por dois pediatras, com a finalidade de verificar a qualidade e veracidade dos dados. Durante todo o estudo, não se encontrou nenhuma entrevista forjada, e o índice kappa (Fleiss, 1981) para o escore de Dubowitz entre os entrevistadores e a revisita foi 0,71, o que garante a qualidade da informação.

O subprojeto de acompanhamento foi concentrado no primeiro ano de vida. Amostras dos nascidos vivos urbanos foram selecionadas, sendo estas crianças acompanhadas ao completarem um, três, seis e 12 meses de ida- de, em seus domicílios, mediante os endereços coletados durante a entrevista hospitalar. As visitas incluíam a aplicação de um questionário e o exame antropométrico das crianças, ambos com metodologia similar à da coorte de 1982 . No primeiro e terceiro meses, tentou-se acompanhar uma amostra de 655 crianças, selecionadas sistematicamente de acordo com o dia de seu nascimento. Para o acompanhamento do sexto e 12o meses, tentou-se localizar uma amostra sistemática de $20 \%$ da coorte, incluindo as crianças visitadas nos acompanhamentos anteriores. Incluíram-se ainda todas as demais crianças nascidas com peso inferior a $2.500 \mathrm{~g}$, pois esse grupo havia sido identificado como de alto risco na coorte anterior. O subprojeto iniciou em fevereiro de 1993 - quando a primeira criança completou um mês - e terminou ao final de dezembro de 1994 - quando a última completou um ano de vida. Os acompanhamentos foram realizados por equipes de entrevistadores das áreas de Nutrição, Enfermagem e Medicina, treinados em técnicas de entrevista e de antropometria. Amostras aleatórias de $5 \%$ das entrevistas foram repetidas por supervisores.

O subprojeto de mortalidade incluiu os óbitos fetais tardios, neonatais e pós-neonatais. Todos os hospitais, cemitérios, cartórios de Registro Civil e a Delegacia Regional de Saúde foram visitados regularmente durante 1993 e 1994 para detectar óbitos de crianças da coorte de 1993. A causa básica do óbito foi identificada através de revisão de prontuários ambulatoriais e hospitalares e de entrevistas com a família e os médicos que assistiram à criança, quando se coletava uma história clínica completa dos eventos que precederam o óbito, baseada numa adaptação dos questionários utilizados na Investigação Inter-Americana de Mortalidade na Infância (Puffer \& Serrano, 1973). Dois árbitros independentes determinaram as causas do óbito, sendo que em caso de discordância estes se reuniam com um terceiro árbitro para a decisão final. As causas eram codificadas conforme a 9a Versão da Classificação Internacional de Doenças (OMS, 1980). Para melhor estudar a mortalidade entre os sete e 364 dias, delineou-se dentro do subprojeto de mortalidade um estudo de casos e controles aninhado na coorte de nascimentos. Para cada óbito, eram selecionadas duas crianças-controle nascidas no mesmo dia, imediatamente antes e após o nascimento do caso. No domicílio, entrevistavam-se então as mães de casos e controles a fim de avaliar fatores de risco, tais como dieta, moradia, cuidados da criança e uso de serviços. 
O subprojeto de morbidade hospitalar incluiu a monitorização de todas as admissões hospitalares de crianças da coorte. De janeiro de 1993 até dezembro de 1994, os hospitais da cidade foram visitados regularmente. A causa da internação era determinada por dois pediatras independentes, com base nas informações do prontuário, da entrevista com as mães e, se necessário, da entrevista com o pediatra. Em caso de discordância, um terceiro árbitro dava o parecer final após reunião conjunta com os dois outros pediatras. Os diagnósticos eram codificados conforme a Classificação Internacional de Doenças (OMS, 1980).

Para investigar os fatores de risco para as internações por doenças infecciosas ocorridas após o primeiro mês de vida, realizou-se um segundo estudo de casos e controles aninhado na coorte. Através de visita domiciliar, as mães dos casos hospitalizados respondiam ao questionário usado no estudo de acompanhamento. Para as crianças internadas com idades entre trinta e 89 dias, usava-se o questionário do acompanhamento de um mês de vida. Para as crianças hospitalizadas com noventa a 179 e 180 a 364 dias, aplicavam-se os questionários dos acompanhamentos dos três e seis meses, respectivamente. O grupo-controle incluiu todas as crianças do subprojeto de acompanhamento.

O subprojeto de desenvolvimento psicomotor incluiu a aplicação do Teste de Denver II (Frankjenburg \& Dodds, 1992) durante os estudos de acompanhamento de um, três, seis e 12 meses. Os entrevistadores foram treinados na administração do teste, utilizando material padronizado, e 5\% das testagens foram repetidas para garantir a qualidade da informação.

\section{Resultados}

A Tabela 1 mostra o número total de crianças incluídas no estudo em cada categoria de renda familiar e de peso ao nascer, para as duas pesquisas. A Tabela 2 mostra o número de nascidos vivos, para as mesmas categorias. Não foram registradas perdas ou recusas no estudo hospitalar de 1982, mas em 1993 foram contabilizadas sete perdas e nove recusas, ou seja, $0,3 \%$ do total.

O número absoluto de nascimentos caiu durante a década, apesar do crescimento na população urbana. O número de nascimentos no grupo de renda mais baixa caiu, havendo aumentado nos grupos de alta renda. Houve redução no número de crianças em todos os grupos de peso ao nascer.
Tabela 1

Número de crianças nascidas vivas nos hospitais de Pelotas conforme a renda familiar e o peso ao nascer, 1982 e 1993.

\begin{tabular}{|c|c|c|c|}
\hline \multirow[t]{2}{*}{ Grupos } & \multicolumn{3}{|c|}{ Número de nascidos vivos } \\
\hline & 1982 & 1993 & $\begin{array}{c}\text { Diferença (\%) } \\
(1993-1982)\end{array}$ \\
\hline \multicolumn{4}{|c|}{$\begin{array}{l}\text { Renda familiar } \\
\text { (salários mínimos) }\end{array}$} \\
\hline$\leq 1$ & 1.288 & 967 & $-321(-24,9 \%)$ \\
\hline $1,1-3$ & 2.789 & 2.148 & $-641(-23,0 \%)$ \\
\hline $3,1-6$ & 1.091 & 1.203 & $112(10,3 \%)$ \\
\hline $6,1-10$ & 382 & 433 & $51 \quad(13,4 \%)$ \\
\hline$>10$ & 335 & 385 & $50 \quad(14,9 \%)$ \\
\hline \multicolumn{4}{|c|}{ Peso ao nascer $(\mathrm{g})$} \\
\hline$<2.500$ & 534 & 510 & $-24 \quad(-4,5 \%)$ \\
\hline $2.500-2.999$ & 1.393 & 1.311 & $-82 \quad(-5,9 \%)$ \\
\hline $3.000-3.499$ & 2.219 & 2.050 & $-169(-7,6 \%)$ \\
\hline$\geq 3.500$ & 1.763 & 1.361 & $-402(-22,8 \%)$ \\
\hline Total & 5.914 (a) & 5.249 (b) & $-665(-11,2 \%)$ \\
\hline
\end{tabular}

(a) Em 1982, não foi possível obter dados sobre a renda familiar para 29 crianças e sobre o peso ao nascer de cinco crianças.

(b) Em 1993, não foi possível obter dados sobre a renda familiar para 113 crianças e sobre o peso ao nascer de 17 crianças.

Tabela 2

Número de crianças nascidas (incluindo natimortos) nos hospitais de Pelotas conforme a renda familiar e o peso ao nascer, 1982 e 1993.

\begin{tabular}{|c|c|c|c|}
\hline \multirow[t]{2}{*}{ Grupos } & \multicolumn{3}{|c|}{ Número de nascidos totais } \\
\hline & 1982 & 1993 & $\begin{array}{c}\text { Diferença (\%) } \\
(1982-1993)\end{array}$ \\
\hline \multicolumn{4}{|c|}{$\begin{array}{l}\text { Renda familiar } \\
\text { (salários mínimos) }\end{array}$} \\
\hline$\leq 1$ & 1.321 & 984 & $-337(-25,5 \%)$ \\
\hline $1,1-3$ & 2.837 & 2.166 & $-671(-23,7 \%)$ \\
\hline $3,1-6$ & 1.105 & 1.217 & $112(10,1 \%)$ \\
\hline $6,1-10$ & 383 & 437 & $54 \quad(14,1 \%)$ \\
\hline$>10$ & 336 & 386 & $50 \quad(14,9 \%)$ \\
\hline \multicolumn{4}{|c|}{ Peso ao nascer (g) } \\
\hline$<2.500$ & 585 & 525 & $-60(-10,3 \%)$ \\
\hline $2.500-2.999$ & 1.407 & 1.312 & $-95 \quad(-6,8 \%)$ \\
\hline $3.000-3.499$ & 2.233 & 2.057 & $-176 \quad(-7,9 \%)$ \\
\hline$\geq 3.500$ & 1.779 & 1364 & $-415(-23,3 \%)$ \\
\hline Total & 6.011 (a) & 5.304 (b) & 707 \\
\hline
\end{tabular}

(a) Em 1982, não foi possível obter dados sobre a renda familiar para 29 crianças e sobre o peso ao nascer de sete crianças.

(b) Em 1993, não foi possível obter dados sobre a renda familiar para 114 crianças e sobre o peso ao nascer de 46 crianças. 
Tabela 3

Percentuais de crianças localizadas nos estudos de acompanhamento conforme a renda familiar e o peso ao nascer, 1982 e 1993.

\begin{tabular}{|c|c|c|c|}
\hline \multirow[t]{3}{*}{ Grupos } & \multicolumn{3}{|c|}{$\begin{array}{l}\text { Percentuais de crianças localizadas } \\
\text { em cada estudo de acompanhamento }\end{array}$} \\
\hline & \multicolumn{2}{|c|}{1982} & \multirow[t]{2}{*}{1993 (a) } \\
\hline & $1 \underline{\text { 으 }}$ & 2으 & \\
\hline \multicolumn{4}{|c|}{ Renda familiar (SM) } \\
\hline$\leq 1$ & 81,0 & 84,6 & 92,3 \\
\hline $1,1-3$ & 84,8 & 89,4 & 92,8 \\
\hline $3,1-6$ & 80,2 & 89,4 & 95,0 \\
\hline $6,1-10$ & 81,3 & 83,3 & 93,3 \\
\hline$>10$ & 82,8 & 83,0 & 95,5 \\
\hline \multicolumn{4}{|c|}{ Peso ao nascer $(\mathrm{g})$} \\
\hline$<2.500$ & 85,2 & 89,0 & 92,2 \\
\hline $2.500-2.999$ & 86,0 & 86,2 & 92,7 \\
\hline $3.000-3.499$ & 81,9 & 87,6 & 94,2 \\
\hline$\geq 3.500$ & 79,7 & 87,4 & 95,1 \\
\hline Total & 82,3 & 87,3 & 93,4 \\
\hline
\end{tabular}

(a) Estudo de acompanhamento dos 12 meses. fatores que facilitaram a realização das pesquisas aqui descritas, encontram-se o alto percentual de nascimentos hospitalares (superior a 99\%), o tamanho médio da cidade estudada, a ausência de grandes fluxos migratórios e a prevalência relativamente baixa de pobreza extrema, se comparada com o restante do país. O tamanho da cidade propicia estudos de coorte por ser, por um lado, suficientemente grande para a ocorrência de um número razoável de eventos (por exemplo, óbitos) e não ser, por outro, uma metrópole com os conseqüentes problemas de alta mobilidade populacional, fluxo imigratório e dificuldades de deslocamento e segurança das equipes de entrevistadores.

Mesmo assim, diversas estratégias tiveram que ser utilizadas para minimizar as perdas de acompanhamento. No primeiro acompanhamento da coorte de 1982, a utilização dos endereços obtidos nos hospitais levou a um índice de perdas de quase $18 \%$, o que - embora ainda razoável no contexto de um estudo brasileiro de uma coorte populacional acompanhada por um ano - colocava em risco os acompanhamentos subseqüentes, nos quais as perdas seriam provavelmente ainda maiores devido ao decurso do tempo. Para o segundo acompanhamento dessa coorte, portanto, optou-se por uma estratégia radicalmente diferente, que consistiu em visitar todos os domicílios urbanos em busca das crianças de 1982, cujas informações seriam posteriormente ligadas aos questionários hospitalares. Isto resultou em serem as perdas do segundo acompanhamento substancialmente inferiores às do primeiro.

Em 1993, optou-se por estudar uma subamostra das crianças com maior freqüência de visitas. Isto impediu o uso do método do censo domiciliar utilizado com sucesso para acompanhar a coorte anterior. A experiência do primeiro acompanhamento de 1982 levou a equipe de pesquisa a ter um cuidado ainda maior na definição dos endereços domiciliares. Durante o estudo perinatal de 1993, solicitavam-se às mães informações detalhadas referentes a seu endereço e sobre como encontrá-lo (linha e parada de ônibus, referências locais), sobre o local de trabalho do pai e mãe, endereço de parentes próximos, telefones para contato e - caso a família pretendesse mudar-se - endereço futuro. Esses cuidados resultaram em um aumento substancial no percentual de crianças localizadas, sendo alcançadas mais de $93 \%$ das crianças após um ano, um índice surpreendentemente alto, mesmo para países desenvolvidos.

Diz um adágio da epidemiologia que "a melhor solução para as perdas é não tê-las”. Sen- 
do isto inviável, a alternativa é pelo menos caracterizar os indivíduos perdidos, para verificar se algum subgrupo populacional estaria sendo deixado de fora, o que poderia distorcer os resultados. Os resultados acima (Tabela 3) mostram haverem sido as perdas relativamente similares nos diferentes grupos de renda familiar e de peso ao nascer, o que sugere ser válido o estudo das associações entre estas variáveis e os desfechos. Embora as diferenças de acompanhamento para os grupos de renda familiar em 1982 tenham sido estatisticamente significativas, isto se deve mais ao grande tamanho da amostra do que à magnitude das taxas de acompanhamento, que variaram no máximo em seis pontos percentuais.

É ainda digna de nota a queda de mais de $10 \%$ no número de nascimentos, apesar do crescimento populacional da cidade. Esse de- clínio evidencia a redução na fecundidade, fenômeno observado em todo o país nas últimas décadas (Frias \& Carvalho, 1994).

O presente artigo demonstrou ser possível realizar em nosso meio estudos de coorte de base populacional. A existência de dois estudos realizados com um intervalo de 11 anos na mesma cidade fornece uma oportunidade única para o entendimento das mudanças ocorridas nos indicadores de saúde materno-infantil e em seus principais determinantes. Os artigos que se seguem nesse suplemento resumirão os principais resultados desta comparação, em termos das características das mães e suas famílias, do peso ao nascer, da mortalidade perinatal e infantil, das hospitalizações, do aleitamento e dieta, do crescimento, do desenvolvimento psicológico e da utilização de serviços de saúde.

\section{Agradecimentos}

Em suas diversas fases, o estudo da coorte de 1982 foi financiado pelo International Development Research Center do Canadá, pela Organização Mundial da Saúde, pelo Overseas Development Administration do Reino Unido, pelo Unicef, pelo Conselho Nacional de Desenvolvimento Científico e Tecnológico (CNPq) e pela Fundação de Amparo à Pesquisa do Rio Grande do Sul.

O estudo da coorte de 1993 foi financiado pela Comunidade Econômica Européia, pela Organização Mundial da Saúde, pelo Conselho Nacional de Desenvolvimento Científico e Tecnológico (CNPq) e pela Fundação de Amparo à Pesquisa do Rio Grande do Sul.

\section{Referências}

BARROS, F. C. \& VICTORA, C. G., 1991. Epidemiologia da Saúde Infantil: Um Manual para Diagnósticos Comunitários. São Paulo: Unicef/Hucitec.

BARROS, F. C.; VICTORA, C. G. \& VAUGHAN, J. P., 1990. The Pelotas birth cohort study, 1982-1987. Strategies for following up 6,000 children in a developing country. Pediatric and Perinatal Epidemiology, 4:267-282.

DUBOWITZ, L. M. S.; DUBOWITZ, V. \& GOLDBERG C., 1970. Clinical assessment of gestacional age in the newborn infant. Journal of Pediatrics, 77:1-10.

FLEISS, J. L., 1981. Statistical Methods for Rates and Proportions. 2nd ed., New York: Wiley.

FRANKENBURG, W. K. \& DODDS, J. B., 1992. The Denver II Developmental Sreening Test. Denver: Denver Developmental Materials.

FRIAS, L. A. M. \& CARVALHO, J. A. M., 1994. Diferenciais da transição dos níveis e padrões de fecundidade. Anais do IX Encontro de Estudos Populacionais.. Vol. 2, pp. 23-46. Belo Horizonte: ABEP.

GRANT J., 1995. Situação Mundial da Infância 1995. Brasília: Unicef. 
JELLIFFE, D. B., 1966. The Assessment of the Nutritional Status of the Community. Geneva: World Health Organization. (WHO, Monograph Series, no 53.)

KLERING, L. R., 1992. Análise do desempenho dos municípios do Rio Grande do Sul em 1991. Análise 3:211-253.

LAURENTI, R.; JORGE, M. H. P. M.; LEBRÃO, M. L. \& GOTLIEB, S. L. D., 1985. Estatísticas de Saúde. São Paulo: Epu/Edusp.

MACEDO, R., 1988. Brazilian children and the economic crisis: the evidence from the state of São Paulo. In: Adjustment with a Human Face. Volume II. Country Case Studies (G.A. Cornia, R. Jolly \& F. Stewart, eds.), pp. 28-56, New York: Unicef.

OMS (Organização Mundial da Saúde), 1980. Classificação Internacional de Doenças. Revisão 1975. São Paulo: Centro da OMS para Classificação de Doenças em Português.

PUFFER, R. R. \& SERRANO, C. V., 1973. Patterns of Mortality in Childhood: the Inter-American Investigation of Mortality in Childhood. Scientific Publication no 262. Washington: Pan American Health Organization.
SSMA (Secretaria da Saúde e do Meio Ambiente), 1995. Estatísticas de Saúde: Mortalidade. Vol. 19, 1993. Porto Alegre: Divisão de Informação em Saúde/Assessoria Técnica e de Planejamento.

UNICEF/IBGE (Fundo das Nações Unidas para a Infância/Fundação Instituto Brasileiro de Geografia e Estatística), 1994. Indicadores Sociais: Crianças e Adolescentes. Edição especial: Censo Demográfico 1991. Rio de Janeiro: IBGE.

VICTORA, C. G.; BARROS, F. C.; HALPERN, R.; MENEZES, A. M. B.; HORTA, B. L.; TOMASI, E.; WEIDERPASS, E.; CÉSAR, J. A.; OLINTO, M. T. A.; GUIMARÃES, P. R. V.; GARCIA, M. M. \& VAUGHAN, J. P. (1996). Estudo longitudinal da população materno-infantil de Pelotas, RS, 1993: aspectos metodológicos e resultados preliminares. Revista de Saúde Pública, 30:34-45.

VICTORA, C. G.; BARROS, F. C. \& VAUGHAN, J. P., 1989. Epidemiologia da Desigualdade: Um Estudo Longitudinal de 6.000 Crianças Brasileiras. 2a ed., São Paulo: Cebes/Hucitec. 